\title{
Das christliche Leben als ein Weg - Elemente der Wallfahrtstheologie
}

\section{Christian life as a way. Selected elements theology of pilgrimage}

\begin{abstract}
The Author presents some Biblical texts on symbolic meaning of a way. In theological meaning a way is a symbol of moving forward, developing, growing in the Christian faith, hope, love and wisdom. Progress in the development of sainthood of a person is of a great significance. In this context, the author refers to pilgrimage which is a symbol of the entire Christian life being approaching God. It covers many religious practices, which help to renew the Christian life, develop faith and love.
\end{abstract}

\section{Keywords}

Pilgrimage, Bible, way, faith, sainthood.

Das Christentum - die Religion des Neuen Testaments wurde in der Apostelgeschichte als „ein Weg “ bezeichnet ${ }^{1}$. Die ersten Christen waren davon überzeugt, dass sie den bisher unbekannten, wahren Lebensweg gefunden haben. Dieser Lebensweg war sowohl die von Christus verkündete Wahrheit, aber vor allem

\footnotetext{
${ }^{1}$ Apg 9,2; 18,25; 24,22.
} 
er selbst ${ }^{2}$. Es kann seltsam wirken, dass sogar die Feinde von Christus eingestanden haben, Christus lehre „wirklich den Weg Gottes“3. Derjenige, der sich den Christen angeschlossen hat, entschied sich für den Beginn einer neuen geistlichen Wanderung. Es verlangte von ihm einen radikalen Wandel des ganzen Verhaltens, besonders der moralischen Einstellung. Von nun an sollte der Anhänger Christi den alten Menschen in sich, der sich nach den Begehren des Fleisches richtet, begraben und sich nach dem Begehren des Geistes, in Gerechtigkeit und Liebe richten ${ }^{4}$. Das Verbleiben auf diesem Weg sollte das Erreichen des endgültigen Lebenszieles, der Erlösung, versichern ${ }^{5}$.

\section{Die Lehre von den zwei Lebenswegen}

In der christlichen Lehre war immer die Lehre von den zwei Wegen sehr lebendig. Sie bildet gewissermaßen eine Zusammenfassung der ganzen evangelischen Moralität ${ }^{6}$ und entstammt noch dem Alten Testament. Ihren Inhalt setzten später die Kirchenväter fort.

Schmal und schwierig ist der Weg, der zum ewigen Leben führt, im Gegensatz zum bequemen und breiten Weg, der zum Verderben führt ${ }^{7}$. Der erste Weg ist auf dem Gehorsam gegenüber den Weisungen des Herrn gegründet ${ }^{8}$. Er wurde als „der Pfad der Gerechten“9, , ,der Pfad der Gerechtigkeit” ${ }^{10}$, „Pfad zum Leben”, „Wege zum Leben“"11, „rechter Weg”" ${ }^{12}$ bezeichnet. Dieser Weg

${ }^{2}$ Vgl. Joh 14,6.

${ }^{3} \mathrm{Mk} 12,14$.

${ }^{4}$ Vgl. Gal 5,16-17.

${ }^{5}$ Vgl. Päpstlicher Rat der Seelsorge für die Migranten und Menschen unterwegs Die Pilgerfahrt zum großen Jubiläum 2000, Nr 11.

${ }^{6}$ Vgl. X. Leon-Dufour, Słownik teologii biblijnej, Poznań 1990, S. 221; M. Lurker, Słownik obrazów i symboli biblijnych, Poznań 1989, S. 46.

7 Vgl. Mt 7,13-14.

${ }^{8}$ Vgl. Ps 1,2 .

9 Spr 4,18.

10 Spr 12,28.

11 Ps 16,11; Apg 2,28.

12 Ps 101,6. 
gründet sich auf der Einhaltung der Gerechtigkeit ${ }^{13}$, der Treue zur Wahrheit ${ }^{14}$, dem Streben nach Frieden ${ }^{15}$. Der Psalmist lobt denjenigen, der „den Herrn fürchtet und der auf seinen Wegen geht ${ }^{\text {“16. }}$. Der zweite Weg ist der Weg der Sünder ${ }^{17}$ und der Hinterlistigen ${ }^{18}$, welche die Gebote Gottes nicht achten. Er führt in den Abgrund ${ }^{19}$, und bringt auf Abwege ${ }^{20}$.

\section{Der Glaube ist ein Weg}

Der Glaube der Kirche und jedes Christen bedeutet etwas Dynamisches, was dem Wechsel unterworfen ist. Man könnte also feststellen, dass der Glaube „unterwegs" ist, das heißt in ständiger Entwicklung. Das Zweite Vatikanische Konzil hat den Glauben mit der Wallfahrt verglichen ${ }^{21}$. Wie Johannes Paul II. schreibt: „Die Wallfahrt des Glaubens deutet von der inneren Geschichte, man kann sagen - von der «Seelengeschichte»" ${ }^{22}$. Durch abwechselnde Lebenserfahrungen und Proben erreicht der Christ einen immer reiferen Glauben, er wird in ihm immer vollkommener und stärkt sich in ihm. Man darf natürlich eine umgekehrte Entwicklung nicht ausschließen - das Nachlassen des Glaubens. Diese Erfahrung zeigt geradezu, dass mancher Gläubige Höhen und Tiefen erlebt. Und auch der Zweifel regt sich in ihm. Es kommt sogar manchmal zum Verrat Christi.

Die christlichen „Schutzpatronen des Glaubens” sind besonders der Patriarch Abraham - "der Vater aller Gläubigen” genannt - und Maria „die Mutter aller Gläubigen“. Ihr Leben zeigt deutlich, dass der Glaube nicht für immer gegeben und gefestigt ist. Ganz im Gegenteil erfordert er immer wieder, neue

\footnotetext{
13 Vgl. Spr 8,20.

${ }^{14} \mathrm{Vgl}$. Tob 1,3.

15 Vgl. Lk 1,79; Röm 3,17.

16 Ps $128,1$.

17 Vgl. Ps 1,1; Spr 4,14.

18 Vgl. Spr 8,13.

19 Vgl. Ps 1,6.

20 Vgl. 2 Petr 2,2.

${ }^{21}$ Bes. Lumen gentium, Kap. VII.

22 Enzyklika Redemptoris Mater, 6.
} 
Entscheidungen zu treffen, Gottes Pläne zu entdecken und sich für den Willen Gottes zu entscheiden. Das christliche Leben sei, laut dem Zweiten Vatikanischen Konzil, das Wandern in Abrahams Glauben ${ }^{23}$. Die Kirche folgt Maria in der Wallfahrt des Glaubens ${ }^{24}$. Der Christ dagegen blickt vor allem auf die Seite von "Jesus, dem Urheber und Vollender des Glaubens"25.

Am Anfang des dritten Jahrtausends erschien der apostolische Brief von Johannes Paul II., in dem er die sich vor der Kirche eröffnende neue „Wegetappe" skizzieren wollte, die geistige Anforderungen stellt ${ }^{26}$. Unter zahlreichen Hinweisen zeigte er als Beispiel das Leben der Jünger, die einen langen und mühseligen Weg durchschritten, bevor sie an den Glauben an Christus und seine Erlösungsbotschaft gelangten. Dieser Weg hat seine Etappen, auf denen der Glaube wächst und sich festigt. Ähnlich war es im Leben des hl. Petrus. Die vollkommene Wahrheit über den Messias erkannte er nicht nur dank seinen eigenen Bemühungen - dank „Fleisch und Blut" - sondern dank der übernatürlichen Gnade, d.h. dank der Offenbarung, die er von seinem himmlischen Vater erhielt ${ }^{27}$. Der hl. Thomas erkannte Christus ebenfalls erst nach innerem Zögern und Zweifeln, als er dem Auferstandenen begegnete und ihn mit seinen Händen berührte ${ }^{28}$. Aber auch im Fall des hl. Thomas beeinflusste ihn nicht nur das Berühren Christi, sondern eher der übernatürliche Glaube, der fähig ist in das Geheimnis der Auferstehung einzudringen. Als Symbol des Wachsens und Reifens im Glauben gilt der Weg nach Emmaus, auf dem die zwei Jünger nicht nur eine in Kilometern messbare Entfernung sondern auch „eine geistige Entfernung “ zurücklegten - sie erreichten die Erkenntnis des Mysterium Christi $^{29}$. Auf dem Weg dahin, die vollkommene Wahrheit über Christus und die Worte seiner Lehre zu verstehen, lernten sie, dass das Beobachten seiner Taten und das Zusammensein mit ihm eine gewisse Bedeutung hatten. Jedoch auch in diesem Fall trug die Gnade Gottes entscheidend zur Einsicht bei ${ }^{30}$.

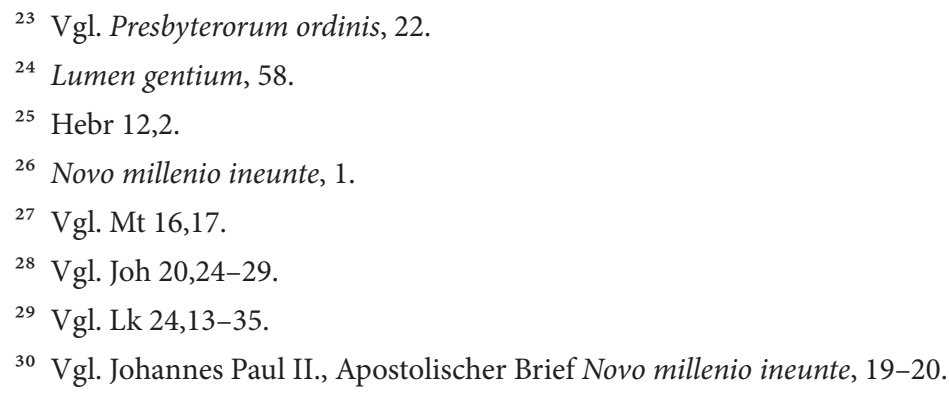


Die Kraft der Gnade Gottes widerspricht keineswegs der Notwendigkeit der menschlichen Bemühungen, die oft einen heroischen Einsatz brauchen. $\mathrm{Zu}$ denselben Jüngern sagte Jesus: „Wer mein Jünger sein will, der verleugne

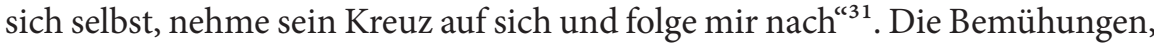
das Sich-Selbst-Verleugnen und die Bereitschaft sich aufzuopfern sind die besonderen Merkmale des Weges, den alle Jünger Christi betreten sollten, die mit ihm den Hügel von Golgatha besteigen wollen, um von den Gnaden des Kreuzes zu schöpfen ${ }^{32}$. Auf diese Weise wird das Leben des Christen zum Weg der Nachfolge Christi.

\section{Hoffnung, Liebe und Weisheit im Kontext des Lebensweges}

Nicht nur der christliche Glaube ist „unterwegs“. Man könnte feststellen, dass das ganze christliche Leben, in all seinen Aspekten, sich im ständigen Wandel und in der Entwicklung befindet. Das Symbol des Weges dient zur Beschreibung des Verlaufs des geistigen Reifens und Wachsens des Christen ${ }^{33}$. Die Hoffnung ist eine besondere Tugend des christlichen Lebensweges. Sie hilft auf dem Weg durchzuhalten und die Schwierigkeiten zu überwinden ${ }^{34}$. Sie lenkt das menschliche Dasein auf die Zukunft. Die übernatürliche Hoffnung führt von dieser begrenzten Welt zum ewigen Leben. Demzufolge enthält sie in sich eschatologische Elemente. Als solche verleiht sie dem irdischen Leben neue, höhere Anregungen ${ }^{35}$. Die Liebe ist der Beweggrund des christlichen Lebensweges. Wir sollten uns auf der Lebenswallfahrt durch die Taten der Liebe gegenseitig unterstützen. Der hl. Paulus ermuntert uns: „liebt einander” oder

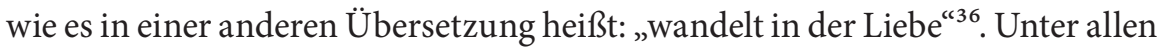
Tugenden hat die Liebe die fundamentale Bedeutung. Man kann sagen, dass

31 Mt 16,24.

${ }^{32}$ Vgl. Die Pilgerfahrt zum großen Jubiläum, zit. Dokum., 9.

${ }^{33}$ Vgl. R. Häselhoff, Sinn Unterwegs. Grundlegendes und Praktisches zur Wallfahrt, Thaur 1999, S. 107.

${ }^{34} \mathrm{Vgl}$. Lumen gentium, 7; Ad gentes divinitus, 5.

${ }^{35}$ Vgl. Gaudium et spes, 21 u 38.

${ }^{36}$ Eph 5,2. 
sie über das Tor der Ewigkeit hinaus reicht. Während alle anderen Tugenden vergehen, wird die Liebe ewig bestehen bleiben ${ }^{37}$.

Die Liebe öffnet uns auf eine andere Person und auf den Dialog mit ihr. Dieser Dialog trägt zu geistiger Entwicklung, sowohl des Gebers als auch des Empfängers, bei. Der Mensch wurde demzufolge von Gott als ein soziales Wesen geschaffen. $\mathrm{Zu}$ seiner Weiterentwicklung benötigt er den anderen Menschen. Indem er den anderen Menschen beschenkt, bereichert er sich geistig selbst. Eine besondere Rolle spielt hier die Tugend der Gastfreundschaft. Die Gastfreundschaft schafft Bindungen zwischen den Ankömmlingen und den Gastgebern, sie ist zugleich eine Gelegenheit, verschiedene Akte der Liebe zu erweisen. Christus identifiziert sich mit jedem Menschen, der Hilfe braucht und lehrt, jeden Menschen in den Personen fremder Ankömmlinge anzunehmen ${ }^{38}$. Der hl. Petrus ermuntert wiederum dazu, das Erweisen der gegenseitigen Gastfreundschaft zum konkreten Zeichen der Liebe werden zu lassen ${ }^{39}$. Wir möchten also an dieser Stelle folgende Wahrheit betonen, die sich seit den Anfängen im Mittelpunkt der christlichen Lehre befand: Durch Liebe, die wir einer zweiten Person erweisen, treffen wir uns mit Christus selbst und dienen ihm selbst in seinen Brüdern.

Indem sich der Mensch auf dem Lebensweg fortbewegt, schöpft er Weisheit. Der alttestamentliche Weise sagt: „Wer viel gereist ist, hat reiches Wissen und der Erfahrene redet verständig “40. Ein Wanderer, der die Welt durchzieht, lernt sie kennen und gewinnt viel Erfahrung. Darum wird die geographische Wanderung eine Gelegenheit zu seiner Entfaltung ${ }^{41}$. Man kann deswegen die Weisheit zu diesen Tugenden rechnen, die besonders mit dem „Unterwegs sein" verbunden sind. Das Zurücklegen des Weges, auf dem der Mensch mit verschieden Lebensproblemen konfrontiert werden kann, die Erfahrung der Größe der Welt und der auf der Welt herrschenden Rechte und zugleich das Erleben seiner eigenen Begrenztheit, erlauben ihm, den Sinn des eigenen Daseins und der uns umgebenen Wirklichkeit tiefer zu erfassen. Der geistige Weg des Menschen ist demnach mit dem Fortschreiten in Weisheit verbunden.

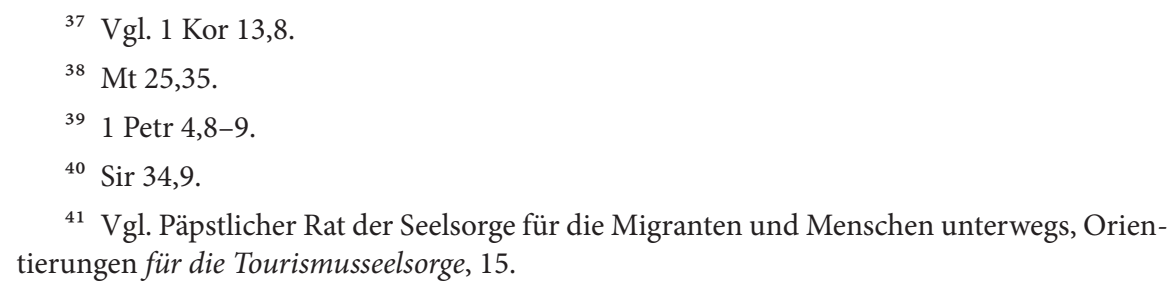

${ }^{41}$ Vgl. Päpstlicher Rat der Seelsorge für die Migranten und Menschen unterwegs, Orientierungen für die Tourismusseelsorge, 15. 
Das Entdecken der Pläne Gottes gegenüber dem Menschen und der Welt trägt hier eine besondere Bedeutung. Wie die Wanderung des Auserwählten Volkes in das Gelobte Land das Fortschreiten zur Erkenntnis des Herren war, so sollte unsere irdische Wallfahrt ein ständiges Fortschreiten zum Verständnis des Willen Gottes gegenüber der ganzen Welt und gegenüber jedem von uns sein. Es ermöglicht dem Christen eine wahre, in Gott verwurzelte Weisheit zu gewinnen. Die vollkommene Gotteserkenntnis wird identisch mit dem Erlangen des Zieles der irdischen Wallfahrt - des Landes ewiger Versprechen und Ruhe ${ }^{42}$.

\section{Der Weg zur Heiligkeit}

So betrachtet können wir feststellen, dass der geistige Weg des Menschen vor allem den Fortschritt im Guten, demnach das Streben nach Heiligkeit bedeutet ${ }^{43}$. Im Katechismus der Katholischen Kirche lesen wir: „Die Schöpfung kam jedoch aus den Händen des Schöpfers nicht ganz fertig hervor. Sie ist so geschaffen, dass sie noch ,,auf dem Weg“ (,in statu viae“) zu einer erst zu erreichenden letzten Vollkommenheit ist, die Gott ihr zugedacht hat" ${ }^{\text {". }}$. Um so mehr sollte der Mensch, die Krone der Schöpfung, zu Heiligkeit, d.h. zur Vollkommenheit in Liebe, heranwachsen ${ }^{45}$. Die Heiligkeit ist notwendig, um die Freude des Himmels zu betreten, demnach das Ende der irdischen Wallfahrt zu erreichen ${ }^{46}$. Der hl. Augustinus fragt: „Was bedeutet geh?“ und antwortet gleich: „Es bedeutet verfahre im Guten.... im wahren Glauben, in guten Sitten"47.

Dieser menschliche Weg bedeutet vielseitige Bemühungen, die unternommen werden, um die Sünde in sich zu überwinden und sich der Heiligkeit zu nähern. Der hl. Petrus ermuntert die Empfänger seines Briefes: „Da ihr Fremde und Gäste seid in dieser Welt, ermahne ich euch: gebt den irdischen Begierden nicht nach,

${ }^{42}$ Vgl. Z. Kijas, Biblijne metafory o miejscu i roli stworzenia we wspólnocie człowieka z Bogiem, in: Między niebem a ziemią. Ku etyce ekologicznej, Hrsg. von A. Dyduch-Falniowska, M. Grzegorczyk, Z. Kijas, Z. Mirek, Kraków 2000, S. 42.

${ }^{43}$ Vgl. I. Dec, „Homo viator” jako kategoria antropologiczna, „Collectanea Theologica” 55 (1985)2, S. 16.

${ }^{44}$ N. 302.

${ }^{45}$ Lumen gentium,16. vgl. Novo millenio ineunte, zit. Dokum., 30-31.

${ }^{46}$ Vgl. Katechizm Kościoła Katolickiego, 1030.

47 Kazanie 256, zit. nach Liturgia godzin, Poznań 1988, Bd. 4, S. 483. 
die gegen die Seele kämpfen “48. Der Christ, der sich dessen bewusst ist, dass er fremd auf der Welt und seine Zeit auf dieser Welt begrenzt sei, bemüht sich seine Momente in Gottesfurcht zu verbringen ${ }^{49}$. Dies bedeutet u.a. den Gehorsam gegenüber dem Willen Gottes und das Vermeiden dessen, was ihn am Erlangen des endgültigen Zieles hindern könnte. Der Weg des Wandels vom alten Menschen in den neuen ist ein dynamischer Prozess, der nie aufhören sollte ${ }^{50}$. Auf dieser Welt können wir aber die beabsichtigte Vollkommenheit nicht erreichen. Kein Mensch „,ist imstande alle seine eigenen Entwicklungsmöglichkeiten zu erschöpfen“"51.

Daher ist der Weg zur Heiligkeit zugleich ein Weg der unaufhörlichen Bekehrung. Johannes Paul II. sagt, das christliche Leben „in statu viae” sei gleichbedeutend mit dem Leben „in statu conversionis” (in ständige Bekehrung $)^{52}$. Jeder Weg sei ein Weg des Wandels, so A. Grün ${ }^{53}$. Das schon oben zitierte Dokument des Apostolischen Stuhles über Wallfahrten knüpft an den Weg des Auszuges des Auserwählten Volkes aus Ägypten ins Gelobte Land an, es spricht über die gegenwärtige Wallfahrt des Neuen Volkes, die erlauben sollte „das Herz von Stein aus eurer Brust zu nehmen und euch ein Herz von Fleisch zu geben” ${ }^{\text {, }}$, indem es „Gerechtigkeit ${ }^{\text {“55 }}$ und „Treue in Liebe ${ }^{\text {56 }}$ verwirkliche.

Eine Widerspiegelung dieses geistigen Weges des Christen, den wir hier betrachten, war seit den Anfängen das Leben der Menschen, die sich in vollkommener Weise Gott opfern wollten. Schon in den ersten Jahrhunderten bildete sich eine Erfahrung des monastischen Lebens aus, welches manchmal als „asketische Migration“ oder „geistiger Exodus“ bezeichnet wird. Die Berufenen gingen an verlassene Orte, um sich vom bisherigen Leben zu entfernen, sie bemühten sich, in Askese zu leben: im Gebet, im Betrachten des eigenen Lebens

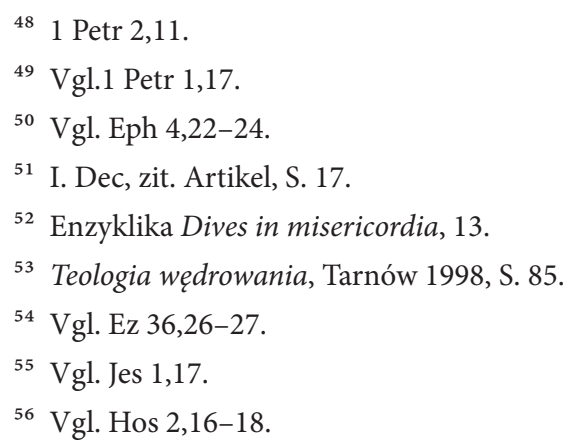


und in der Beherrschung der eigenen Schwächen, um sich der Heiligkeit und Gott zu nähern. Andererseits kennen wir aus der Geschichte Wallfahrten zu den Einsiedlern, die von den Christen als Lebensmeister angesehen wurden. An ihrer Seite wollten sie nach Rat suchen und evangelische Weisheit gewinnen.

Die asketische Migration knüpfte an die alttestamentliche Tradition an, z.B. an den Propheten Elija ${ }^{57}$. Der Auszug in die Wüste sollte sowohl der Begegnung mit Gott, als auch dem geistigen Kampf mit dem Bösen dienen ${ }^{58}$. Die Wüste hat in der biblischen Tradition eine vielseitige Symbolik. Einerseits ist sie ein Ort der Flucht, andererseits ein Ort der Gottes Theophanie und der Begegnung mit Gott. Sie bildet einen Raum der Probe wie auch der besonderen Fürsorge Gottes ${ }^{59}$. Das Verlassen der Alltagsprobleme, das Sich - Verschließen in der Einsiedelei oder im Kloster sollte neue Bedingungen für die Entwicklung des mystischen Lebens schaffen. Einerseits bedeutet es das Zurückziehen von der Welt, andererseits das Betreten des eigenen Inneren, wie der hl. Augustinus schrieb: „nicht um sich in sich selbst einzuschließen, aber um sich selbst zu überschreiten“, um „in sein Herz, in dem die Wahrheit wohnt, eindringen “60. Dieser Weg bedeutet demzufolge eine „Fluchtwallfahrt" und zugleich einen „Weg zur Heiligkeit“. Den Mönchen ging es nicht nur um die Wallfahrt zu einem bestimmten Wallfahrtsort (Wallfahrtszentrum), aber auch um den Übergang von den Alltagsproblemen zu geistigen Angelegenheiten - zur Nähe mit Gott in Gebet und Meditation, die auf dieser Welt eine Antizipation des Himmels bedeuten. „Die Vergöttlichung des Menschen ist das endgültige Ziel der langen geistigen Wallfahrt“. In den Vordergrund der ",asketischen Migration“ rückt demnach der „ekstatische Aspekt ${ }^{\text {“61 }}$. Der berühmte Klostergründer, der hl. Bernhard von Clairvaux meinte, das himmlische Jerusalem, das endgültige Ziel der irdischen Wallfahrt, sei schon anwesend im christlichen Kloster. Man könne es durch das Praktizieren radikaler und tiefer Frömmigkeit erlangen ${ }^{62}$.

57 Vgl. 1 Kön 17,2f.

${ }^{58}$ Vgl. ebd., 13.

59 Vgl. S. Hałas, Pustynia miejscem próby i spotkania z Bogiem. Wybrane zagadnienia z biblijnej teologii pustyni, Kraków 1999, S. 189f, $235 f$.

${ }^{60} \mathrm{H}$. Augustinus, De vera religione 39, 72.

${ }^{61}$ Vgl. Die Pilgerfahrt zum großen Jubiläum, zit. Dokum.; es führt u.a. den hl. Augustinus, den hl. Hieronymus und den hl. Gregor von Nyssa an.

${ }^{62}$ List do biskupa miasta Lincoln, 64.2. zit. nach Die Pilgerfahrt zum großen Jubiläum, zit. Dokum., 14. 


\section{Der Weg des Pilgers als eine Widerspiegelung des Lebensweges}

Die Tradition spricht den Wallfahrtspraktiken eine große Bedeutung zu, die als asketische Mittel zur Bildung der evangelischen Haltungen gelten ${ }^{63}$. Die Wallfahrt bildet eine besondere Art von Formung der christlichen Persönlichkeit. Sie wird zu einem wahren geistigen Weg. Eine deutliche Darstellung dieses Phänomens waren die Andachten zum Großen Jubiläum des Jahres 2000. Zu den wesentlichen Erlebnissen gehörten die Wallfahrten nach Rom und in viele Jubiläumskirchen auf der ganzen Welt. Jedoch im Pilgern, wie es Johannes Paul II. so oft betonte, ging es nicht nur um eine Reise im geographischen Sinne. Als erstes Ziel dieser Art von religiösen Praktiken galt der tiefe geistige Wandel. Jede Wallfahrt sollte für ihre Teilnehmer zu großen Exerzitien werden, und das Erhalten des Jubiläumsablasses ein Weg zu radikaler Bekehrung. Das Überschreiten der Heiligen Pforte der römischen Basilika sollte zu einem Symbol des Übergangs des Christen von der Sünde zum Leben in Gottes Gnade werden ${ }^{64}$. Das Dokument über das Pilgern im Jahr des Großen Jubiläums hebt besonders hervor: „Dieser Weg sollte nicht nur durch den Raum verlaufen, aber vor allem durch das Innerste und durch das menschliche Leben“. Als Frucht dieses Weges gilt die Verzeihung und Versöhnung, die Erneuerung der menschlichen Würde, die Gestaltung sozialer Solidarität und Bildung einer wahren, brüderlichen zwischenmenschlichen Gemeinschaft. Der Pilgerweg drückt es nicht nur symbolisch aus, aber er ist wirklich der Weg zu persönlicher Heiligkeit. Durch das Wachen, Fasten und Gebet bewegt sich der Pilger auf dem Weg der christlichen Vollkommenheit fort und bemüht sich, dank der Gnade Gottes zum „vollkommenen Menschen zu werden und Christus in seiner vollendeten Gestalt darstellen (Eph 4, 13)"65.

Jede Wallfahrt ist mit bestimmten Schwierigkeiten und Verzichten ihrer Teilnehmer verbunden. Sie erfordert Selbstverleugnung und das Übernehmen vielseitiger Begrenzungen. Sie spiegelt den inneren Weg des Glaubens wider, der mit der Bewältigung vieler geistiger Hindernisse, besonders dem Kampf mit der Sünde, verbunden ist. Hier bestätigen sich in spürbarer Weise die Worte

\footnotetext{
${ }^{63}$ Świętość. Dar i zadanie, w: II Polski synod plenarny (1991 - 1999), Poznań 2001, 13.

${ }^{64}$ Bulle Incarnationis mysterium, zit. Dokum., 7-9.

${ }^{65}$ Incarnationis mysterium, zit. Dokum., 7.
} 
Christi: „Wer mein Jünger sein will, der verleugne sich selbst, nehme sein Kreuz auf sich und folge mir nach" ${ }^{36}$. In symbolischer Weise wird diese Wahrheit in der Kreuzwegandacht ausgedrückt, die in vielen Wallfahrtsorten sehr populär ist. Diese Andacht ist eine Art „kleiner Wallfahrt“ - das Mitgehen auf dem Weg Christi. Das Nachdenken über den Leidensweg Christi ermuntert die Christen $\mathrm{zu}$ einer tieferen Betrachtung seines persönlichen Lebens und seiner Treue zu den evangelischen Grundsätzen. Neben der Bußbedeutung sollte sie auch zur Bereitschaft für den moralischen Wandel führen ${ }^{67}$.

Der Pilger bewegt sich zum Wallfahrtsort fort, der ein Symbol des endgültigen Lebenszieles und der Gemeinschaft mit Gott in Ewigkeit ist. Er erwartet dort reichliche Gnaden Gottes zu erhalten. Dieser Wunsch treibt ihn sich fortzubewegen. Es entfaltet und stärkt sich seine Hoffnung, nicht nur im irdischen Sinne verstanden - das Erreichen des Zieles, d.h. der Heiligen Stätte und die Erholung nach der langen Reise, aber auch die übernatürliche Hoffnung - das Erreichen des endgültigen Hauses, d.h. des Himmels, wo er für immer in Gott ruhen wird ${ }^{68}$.

Die Tugend der Nächstenliebe und die aus ihr entspringende Tugend der Gastfreundschaft sind es, die eine große Bedeutung beim Pilgern tragen ${ }^{69}$. Während der Wallfahrt kommt es zu zahlreichen Gelegenheiten sich gegenseitig zu helfen und konkrete Werke der Liebe zu leisten. Auch deswegen, weil sich der Pilger außerhalb des eigenen Hauses befindet, unter Fremden, ohne Unterstützung der ihm nahen Umgebung. Unterwegs werden vielseitige Bindungen geknüpft: zwischen den Pilgern selbst und zwischen den Pilgern und ihren Gastgebern. Es ist eine gute Möglichkeit zum gegenseitigen Austausch und zur Bereicherung durch neue Werte, die jeder besitzt. Sowohl das „zu Gast bitten“ als auch das „zu Gast werden“ wird eine Gelegenheit zur besonderer Gemeinschaft mit Gott, der im anderen Menschen anwesend ist. Jedes Werk der Liebe, wie wir schon früher betonten, ist eine Gelegenheit zum Erweisen der Liebe zu Christus in seinen Brüdern. Indem wir demzufolge den geographischen Raum

${ }^{66} \mathrm{Mt} 16,24$.

67 Vgl. R. Häselhoff, Sinn Unterwegs. Grundlegendes und Praktisches zur Wallfahrt, S. 120121.

68 Vgl. Die Pilgerfahrt zum großen Jubiläum, zit. Dokum., 43; vgl. 1 Thess 4, 17.

69 Vgl. Orientierungen für die Tourismusseelsorge, zit. Doment., 19. 
durchschreiten, öffnet sich vor uns eine Möglichkeit, den geistigen Weg des Wachsens in Liebe zu Gott und zum Nächsten durchzugehen ${ }^{70}$.

Der Pilger begibt sich demnach nicht nur auf den geographischen Weg - von seinem Haus zur Heiligen Stätte, er begegnet vielmehr auch Christus, der für ihn selbst zum Weg wird. Zugleich „wird er in Bewegung gesetzt und zum Vater geführt $^{\text {"71 }}$. Das tiefe und authentische Erleben der Wallfahrt wird für ihn zum Anfang eines radikalen Wandels, zu einer neuen Ausrichtung des Lebens und zum Aufbau einer neuen Beziehung zu Gott und den Nächsten. Sein Glaube entwickelt sich, seine Hoffnung festigt sich und seine Liebe stärkt sich.

\section{Deutsche Übersetzung: Marta Krzanowska} Überprüft: prof. Michaela Hastetter

\section{Bibliography}

Darrieutort A., Droga, in: X. Leon-Dufour, Słownik teologii biblijnej, Poznań 1990, S. 220-222.

Dec I., „Homo viator” jako kategoria antropologiczna, „Collectanea Theologica” 55 (1985) 2, S. 16.

Grün A., Teologia wędrowania, Tarnów 1998.

Hałas S., Pustynia miejscem próby i spotkania z Bogiem. Wybrane zagadnienia z biblijnej teologii pustyni, Kraków 1999.

Häselhoff R., Sinn Unterwegs. Grundlegendes und Praktisches zur Wallfahrt, Thaur 1999. Johannes Paul II., Apostolischer Brief Novo millenio ineunte, 6 Januar 2001.

Johannes Paul II, Enzyklika Redemptoris Mater, 25. März 1987.

Johannes Paul II. Über die Pilgerfahrt zu den Stätten, die mit der Heilsgeschichte verbunden sind http://www.vatican.va/holy_father/john_paul_ii/letters/documents/hf_jp-ii_ let_30061999_pilgrimage_ge.html (30.12.2014).

${ }^{70}$ Vgl. R. Häselhoff, R. Häselhoff, Sinn Unterwegs. Grundlegendes und Praktisches zur Wallfahrt, S. 123.

${ }^{71}$ A. Grün, Teologia wędrowania, Tarnów 1998, S. 85. 
Kijas Z., Biblijne metafory o miejscu i roli stworzenia we wspólnocie człowieka $z$ Bogiem, in: Między niebem a ziemia. Ku etyce ekologicznej, Hrsg. von A. Dyduch-Falniowska, M. Grzegorczyk, Z. Kijas, Z. Mirek, Kraków 2000, S. 29-58.

Lurker M. L., Słownik obrazów i symboli biblijnych, Poznań 1989, S. 45-47.

Päpstlicher Rat der Seelsorge für die Migranten und Menschen unterwegs, Die Pilgerfahrt zum großen Jubiläum 2000, 25 April 1998

http://www.vatican.va/roman_curia/pontifical_councils/migrants/documents/rc_pc_ migrants_doc_19980425_pilgrimage_ge.htm (30.12.2014).

Päpstlicher Rat der Seelsorge für die Migranten und Menschen unterwegs, Heilige Stätten - Erinnerung, Gegenwart und Prophezeiung des lebendigen Gottes, 8. Mai 1999 http://www.vatican.va/roman_curia/pontifical_councils/migrants/documents/ rc_pc_migrants_doc_19990525_shrine_it.html (30.12.2014).

Päpstlicher Rat der Seelsorge für die Migranten und Menschen unterwegs, Orientierungen für die Tourismusseelsorge 29 Juni 2001

http://www.vatican.va/roman_curia/pontifical_councils/migrants/documents/rc_pc_ migrants_doc_20010711_pastorale-turismo_ge.html (30.12.2014). 